\title{
Trauma courses for senior house officers
}

Sir

We read with interest of the success of the Accident and Emergency Department in Sunderland in organizing a trauma course for SHOs based on ATLS principles.

Although we do not have a group of ATLS instructors, we have used the three ATLS trained A\&E staff to provide very similar training to that provided in Sunderland. However, our programme differs in some respects, which may be of interest to readers.

On the principle that efficient and effective resuscitation in the A\&E department depends on a coordinated response of both doctors and nurses, we have organized lectures on ATLS principles for both groups and then invited the nursing staff to take an active role in the moulage situations - something which they have been keen to do.

'Patients' for the moulage have been provided by local resources - two A\&E Registrars suitable disguised, and equipment loaned from the resuscitation room. We have been pleased by the realistic result achieved with simple means.

A video recording is taken of the scenario and this can be played back during the de-briefing session, enabling fatal errors and errors in assessment to be recognized and discussed.

In addition we have made a short video demonstrating intercostal drainage, crycothyroidotomy and central line insertion, with the assistance of the Nottingham University Audio-Visual Department.

There is no suggestion that these courses should in any way be a substitute for the ATLS Provider Course, but we have found them a very useful adjunct to ouve SHO teaching programme and hope that others will be encouraged to experiment in similar techniques.

M. K. GREENE \& R. K. RODEN

Senior Registrar \& Registrar

Accident and Emergency Department

Derbyshire Royal Infirmary

Derby 